\title{
The Extended Hubbard Model Applied to Phase Diagram and the Pressure Effects in $\mathrm{Bi}_{2} \mathrm{Sr}_{2} \mathrm{CaCu}_{2} \mathrm{O}_{8+y}$ Superconductors
}

\author{
E. V. L. de Mello \\ Departamento de Física, Universidade Federal Fluminense, \\ Av. Litorânia s/n, Niterói, R.J., 24210-340, Brazil
}

Received 4 June, 1999. Revised version received on 2 August, 1999

\begin{abstract}
We use the two dimensional extended Hubbard Hamiltonian with the position of the attractive potential as a variable parameter, within a BCS type approach, to study the interplay between the superconductor transition temperature $T_{c}$ and hole content for high temperature superconductors. This method gives some insight on the range and intensity of the Cooper pair interaction. It suggests why different compounds have different values for their measured coherence lengths, and describes the experimental results of the superconducting phase diagram $T_{c} \times n$. The calculations may also be used to study the effect of the applied pressure with the assumption that it increases the attractive potential which is accompanied by an increase in the superconductor gap. In this way we obtain a microscopic interpretation for the intrinsic term and a general expansion for $T_{c}$ in terms of the pressure which reproduces well the experimental measurements on the $\mathrm{Bi}_{2} \mathrm{Sr}_{2} \mathrm{CaCu}_{2} \mathrm{O}_{8+y}$ superconductors.
\end{abstract}

\section{Introduction}

In order to understand the superconductivity mechanism for high- $T_{c}$ superconductors (HTSC), many experiments have attempted to find out possibles correlations between the superconductivity temperatures $T_{c}$ and some physical parameter. Some of these experiments deal with the interplay among $T_{e}$, hole contents, and pressure effects, which have revealed interesting features as the maximum attained $T_{c}[1]$ and have attracted a lot of attention as it is summarized by some review articles[2, 3, 4]. On the other hand, neither one of the several microscopic theoretical models have been able to deal successfully with all these observed basic properties and therefore the nature of the condensate pair has not yet been established. As a consequence, some phenomenological approaches have been advanced in an effort to account for the large experimental data on HTSC. For instance, the $T_{c}$ dependence on the number of carriers per $\mathrm{CuO}$ follows the so-called universal inverse parabolic curve, i.e., $T_{c}=T_{c}^{\max }\left[1-\eta\left(n-n_{o p}\right)^{2}\right]$ where $n_{o p}$ is the optimum carrier density $n$ and $\eta$ is an adjustable parameter which depends on the type of compound.

Recently we have introduced an intermediate approach[5] which is neither pure microscopic nor pure phenomenological. It starts with the microscopic extended Hubbard Hamiltonian ( $\mathrm{t}-\mathrm{U}-\mathrm{V}$ ) on a square lattice of side $a$,

$$
H=-\sum_{\langle i j\rangle, \sigma} t\left(c_{i \sigma}^{\dagger} c_{j \sigma}+\text { h.c. }\right)+U \sum_{i} n_{i \downarrow} n_{i \uparrow}-V \sum_{\langle i j\rangle} n_{i} n_{j}
$$

where $t$ is the nearest neighbor hopping integral, U is the on-site repulsion and $\mathrm{V}$ a phenomenological attractive interaction. Several authors have argued that this Hamiltonian accounts for the physics of the charge carriers $[6,7]$ of HTSC. The strength of the phenomenological potential coupling constants are determined $a$ 
posteriori in comparison with the experimental results for $T_{c} \times n$. The hopping integral $t$ is obtained by comparing with band structure calculations[7]. Using the BCS method and the Fourier transform of the potential we have derived zero and finite temperature gap equations which are integrated up to region where pair formation has a non-negligible probability. In this way we have been able to relate $T_{c}$, hole content $n$ and the strength of the zero temperature gap $\Delta(0)$ as described in detail in Ref.[5]. As concerns the phase diagram, its optimal doping is very sensitive if we move $\mathrm{V}$ from its original nearest neighbor position for the

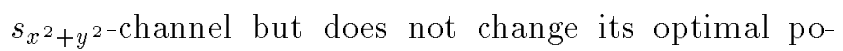
sition for $d_{x^{2}-y^{2}}$-wave. A study of these and others channels as well as their complex mixtures and interplay as function of the position of $\mathrm{V}$ will be published elsewhere[8]. This method was successfully applied to the experimental measurements of the curves $T_{c} \times n$ for the $L a(S r, B a) C u O$ and $Y B a C u O$ compounds[5]. On the other hand if we study the typical parameter which characterizes the strength of the attractive interaction for a square well in $2 \mathrm{D}$ with range $d$ and strength[9] $\mathrm{V}$, $\chi=m_{e} d^{2} V / 2 \hbar^{2}$, we see that for the above two compounds and $\mathrm{Hg} 1201$ and $\mathrm{Bi}_{2} \mathrm{Sr}_{2} \mathrm{CaCu}_{2} \mathrm{O}_{8+y}$, it varies between $10-3000$ and such high values indicate that the size of the bound states (or Cooper pairs) is related to the position of the minimum and the range of the attractive potential. This is the main motivation to change the position of the attractive potential $\mathrm{V}$ from the usual nearest neighbor and it is very interesting to find out that the curves for $T_{c} \times n$ yield

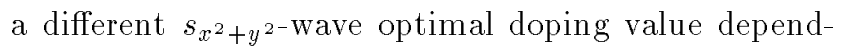
ing on the size of $\mathrm{V}$ while it remains fixed for $d_{x^{2}-y^{2-}}$ wave. Another interesting point from the theoretical point of view is that it provides a natural interpretation for the order parameter expansion introduced by the spin-fluctuation theory[10]. In this approach

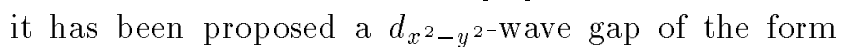
$\Delta_{0}(\vec{k})\left(\cos k_{x} a-\cos k_{y} a\right)$ where $\Delta_{0}(\vec{k})$ is expanded in powers of $\left(\cos _{x} a+\cos k_{y} a\right)$. Analising this expansion, one can easily verify that it contains terms like $\left(\cos _{x} a-\cos k_{y} a\right)\left(\cos k_{x} a+\cos k_{y} a\right)$ which are proportional to $\left(\cos \left(2 k_{x} a\right)-\cos \left(2 k_{y} a\right)\right)$ and what can be seen as a type of $d$-wave gap symmetry that arises from a potential like $V(\vec{k})=V_{0}\left(\cos \left(2 k_{x} a\right)+\cos \left(2 k_{y} a\right)\right)$. By the same token, we can find terms proportional to $\left(\cos \left(3 k_{x} a\right)-\cos \left(3 k_{y} a\right)\right)$ which can be originated by a potential $V(\vec{k})=V_{0}\left(\cos \left(3 k_{x} a\right)+\cos \left(3 k_{y} a\right)\right)$ and so on. Based on this result, we adopt below a notation $V(\vec{k})=V_{0}\left(\cos \left(b k_{x} a\right)+\cos \left(b k_{y} a\right)\right)$, with $b=1,2,3, \ldots$ As one expects, the more distant is the position of $\mathrm{V}$, that is the larger is $b$, smaller is the value of the density necessary to start the superconductivity process of pair formation. In this way we have derived $T_{c} \times n$ curves $[5]$ that agree well with the experimental data when $V$ was placed at $6^{\text {th }}$ (s-wave) and $3^{\text {rd }}$ (d-wave) neighbors for $L a(S r, B a) C u O$ and $Y B a C u O$ compounds respectively which by the way is very close the ratios of their measured coherence length $\xi[11]$. Thus the method provides a very interesting and direct interpretation for the measured values of the coherence length for the HTSC compounds besides the fact that it gives a hint for the superconducting fundamental interaction in HTSC.

We have also extended this approach to study the pressure effects[12] on HTSC. We have noticed that $T_{c}(n)$ is very sensitive to changes in the zero temperature gap amplitude $\Delta(0)$ which is used as an adjustable parameter. Consequently we have introduced the assumption that the pressure induces changes on the attractive potential end ultimately on $\Delta(0)[12]$. This assumption together with the well documented fact that the applied pressure induces a charge transfer from the reservoir layers to the $\mathrm{CuO}_{2}$ planes, has enabled us to derive an expansion for $T_{c}(n, P)$ in power of the pressure. This expansion was applied to the $\mathrm{Hg} 1201,1212$ and 1232 compounds and has reproduced the measured values of $T_{c}$ for a large set of hole content and applied pressures up to $45 \mathrm{GPa}[12]$. We should stress that since the principal interaction mechanism which forms Cooper pairs has yet to be identified, the connection between $T_{c}$, hole content, pressure effects and a microscopic Hamiltonian should provide some clues for this interaction and it is a efficient method to obtain information on the microscopic mechanism for HTSC. Thus our study provides a guide to the strength and range of this fundamental mechanism.

In this paper we shall clarify and illustrate the main physical ideas of the method mentioned above and to show that it is general and can be applied to other HTSC than the Hg-based compounds, as we apply it to the experimental phase diagram and pressure data on Bi2212. The $\mathrm{Bi}_{2} \mathrm{Sr}_{2} \mathrm{CaCu}_{2} \mathrm{O}_{8+y}$ family has been studied by several groups and in particular, we shall discuss firstly the measurements of the dependence of the critical temperature $T_{c}$ on the hole content $n[13]$ to set the pertinent parameters to be used afterwards on the study of the variation of $T_{c}$ under applied external pressures[14]. The $\mathrm{Bi}_{2} \mathrm{Sr}_{2} \mathrm{CaCu}_{2} \mathrm{O}_{8+y}$ is strongly anisotropic, its resistivity on the ab plane is $10^{4}$ larger than its similar along the c-axis[14] which justify the use of a 2D model and has one of the shortest coherence length[11] among all cuprates superconductors and this property will be taken into account to set the parameter $b$ in our calculations. 


\section{The Method}

Following the BCS method[15] we use a many-body wave function which describes the formation of pairs of charge carriers

$$
|\Phi\rangle=\prod_{\vec{k}}\left(u_{\vec{k}}+v_{\vec{k}} c_{\vec{k} \uparrow}^{\dagger} c_{-\vec{k} \downarrow}\right)\left|\Phi_{0}\right\rangle,
$$

where $\left|\Phi_{0}\right\rangle$ is the empty band state and $u_{\vec{k}}^{2}+v_{\vec{k}}^{2}=1$.

In connection with the Hamiltonian of Eq. (1), and the variational approach[15], we obtain

$$
\Delta_{\vec{k}}=-\sum_{\vec{l}} V_{\vec{k} \vec{l}} u_{\vec{l}} v_{\vec{l}}
$$

where $V_{\vec{k} \vec{l}}$ is the Fourier transform of the potential of Eq. (1), and following along the lines of Ref.[7] may be written in a "separable" form, $V_{\vec{k} \vec{l}}=$ $U-2 V\left(\cos \left(k_{x} a\right) \cos \left(l_{x} a\right)+\cos \left(k_{y} a\right) \cos \left(l_{y} a\right)\right)$. A similar approach which takes next-nearest neighbor hopping into account was developed by Angilella et al[7] with the same purpose to study pressure effects on $\mathrm{Bi}_{2} \mathrm{Sr}_{2} \mathrm{CaCu}_{2} \mathrm{O}_{8+y}$ compounds but they only deal with nearest neighbor attractions. With the use of the expressions for $u_{\vec{l}}$ and $v_{\vec{k}}$, we get an equation for the zero temperature gap,

$$
\Delta_{\vec{k}}=-\sum_{\vec{l}} V_{\vec{k} \vec{l}} \frac{\Delta_{\vec{l}}}{2\left(\xi_{\vec{l}}^{2}+\Delta_{\vec{l}}^{2}\right)^{1 / 2}} .
$$

Where $\xi_{\vec{k}}=-2 t\left(\cos \left(k_{x} a\right)+\cos \left(k_{y} a\right)\right)-\mu$,

Thus since the gap has the same functional form of the potential, namely, $\Delta_{\vec{k}}=\Delta(0)\left(\cos \left(k_{x} a\right) \pm\right.$ $\left.\cos \left(k_{y} a\right)\right) / 2$, where the plus sign is for the s-wave and the minus sign is for $\mathrm{d}$-wave channel. The chemical potential $\mu$ yields the density $n$ that must be calculated self-consistently[6] but as it concerns the superconducting phase boundary it suffices to approximate it by the $T=0 \mathrm{~K}$ value of the maximum energy (concentration dependent) in the tight-binding band.

In order to study pair formation, we calculate the probability of finding a hole pair, that is, the condensation amplitude $F_{\vec{k}} \equiv u_{\vec{k}} v_{\vec{k}}$. It is a general result[15] that it has a maximum at $k_{F}$ and drops very rapidly for $|k|>k_{F}$. According to these considerations and taking $\vec{k}=(0,0)$ in Eq. (4), we obtain for s-wave

$$
A=\int_{0}^{\alpha_{\Delta}} \int_{0}^{\beta_{\Delta}} d \alpha d \beta \frac{\left(U-2 V f_{+}(b \alpha, b \beta)\right) f_{+}(b \alpha, b \beta) / 2}{\left(\left(2 t\left(f_{+}(\alpha, \beta)-\mu\right)\right)^{2}+\left(\Delta(0) f_{+}(b \alpha, b \beta) / 2\right)^{2}\right)^{1 / 2}},
$$

where $f_{ \pm}(\alpha, \beta) \equiv \cos \alpha \pm \cos \beta$ and $\alpha=k_{x} a$ and $\beta=k_{y} a . \mu=f_{+}\left(\alpha_{F}, \beta_{F}\right)$ and $\alpha_{F}$ and $\beta_{F}$ are the maximum $T=0$ occupied values (like a Fermi momentum) that depend on the density of holes $n$. The parameter $b$ is used to set the position of the attractive potential $\mathrm{V}$, thus $b=2$ for third nearest neighbor and so on We use $\alpha_{F}=\beta_{F} \approx \operatorname{arcos}(1-n)$ and $\mu=-2 t\left(\cos \left(\alpha_{F}\right)\right)$. The integrations are performed up to $\alpha_{\Delta}$ and $\beta_{\Delta}$, which are determined at values where the condensation amplitude becomes very small, namely, $F_{\vec{k}} \approx 0.01$. This is usually attained for $\xi_{\vec{k}}>6 \Delta(0)$.

For $T \neq 0$, the excitations with their respective probability must be taken into account. The derivation of a self-consistently temperature-dependent gap equation is analogous to that which leads to Eq. (5). At this point, we again follow the BCS approach[15] and assume that $\Delta(T)$ vanishes at the critical temperature $T_{\epsilon}$, which yields the following equation for s-wave symmetry,

$$
B=\int_{0}^{\alpha_{\Delta}} \int_{0}^{\beta_{\Delta}} d \alpha d \beta \frac{\left(U-2 V f_{+}(b \alpha, b \beta)\right) f_{+}(b \alpha, b \beta) \tanh \left(\frac{2 \xi_{\alpha} \beta}{2 K_{B} T_{c}}\right)}{2 t\left(f_{+}(\alpha, \beta)-f_{+}\left(\alpha_{F}, \beta_{F}\right)\right)},
$$

where we again integrate up to $\alpha_{\Delta}$ and $\beta_{\Delta}$

Now, if we had integrated Eqs. (5 and 6) over the whole Brillouin Zone and solve them self-consistently, we would get $A=B=-(2 \pi)^{2}$. Instead of this procedure, we find, for a given density $n$, the value of $T_{c}$ which is compatible, i.e., make the equality $A=B$ for 
a given $\Delta(0)$ and $b$. This is the basic procedure of our method. The same approach can be carried out for $\mathrm{d}-$ wave symmetry but in this case we take $\vec{k}=(0, \pi / 2)$ in Eqs.(5 and 6). Thus, with only one given value for the parameter $b$ and another one for $\Delta(0)$, we can find the value of $T_{c}$ for a given density of carriers $n$ and therefore obtain the curve $T_{c} \times n$.

\section{Pressure Effects}

Now we turn our attention to the study of the HTSC under pressure. One of the effects of the pressure which is generally accepted and well documented in certain materials is an increase of the carrier concentration on the $\mathrm{CuO}_{2}$ planes transferred from the reservoir layers[2, 3, 4]. Such pressure induced charge transfer (PICT) has been confirmed by Hall effect and thermoelectric power measurements on several compounds[3]. Therefore this effect combined with an assumption of an intrinsic variation of $T_{c}$ (linear on the pressure) independently of the charge transfer was largely explored to account for the quantitative relation between $T_{c}$ and the pressure $\mathrm{P}$ and it gave origin to many models[16, 17, 18, 19, 20]. However, the various pressure data taken with the mercury family of compounds $[21,1]$ for underdoped and overdoped compounds of $\mathrm{HgBa}_{2} \mathrm{CuO}_{4-\delta}(\mathrm{Hg} 1201)$ has some features that could not be interpreted $[21,1]$ by these pure charge transfer models by a single set of parameters. On the other hand we could explain the $\mathrm{Hg}$-family data using a single set of parameter and this is an achievement of our method described below.

The study of $T_{c} \times n$ which is necessary to find the appropriated values of $b$ and $\Delta(0)$ (at $P=0$ ) demonstrated the direct proportionality between $\Delta(0)$ and $T_{c}$ which varies with the hole content $n$ and it is maximum at the optimal doping. Taking this result into account and the discussion of the above paragraph, we are led to propose that the effects of pressure are twofold: (i)- The well accepted PICT and an additional assumption; (ii)- A change in the attractive potential $\mathrm{V}$ as proposed previously by Angilella et al[7] which also implies on a change in the zero temperature gap $\Delta(0)$. The assumption "ii" came from the analysis of the curves $T_{c} \times n$ which have revealed that $T_{c}$ is proportional to $\Delta(0)$ but with a large constant of proportionality around the optimal doping[12] $n_{o p}$ and smaller near the both underdoped and overdoped extremes of the $T_{c} \times n$ curve. The quantitative changes on $\mathrm{V}$ due to the applied pressure were estimated in Ref.[7] for the $\mathrm{Bi}_{2} \mathrm{Sr}_{2} \mathrm{CaCu}_{2} \mathrm{O}_{8+y}$ using the measured values[14] of the compressibility tensor under the linear approximation and the self-consistently equations.

Thus the PICT (i) implies that $n(P)=n+\Delta n(P)$ and the assumption of a pressure dependent gap (ii) implies that $\Delta(0, P)=\Delta(0)+\Delta \Delta(0, P)$ and both equations lead to $T_{c}(n, P)=T_{c}(n(P), \Delta(0, P))$. Therefore to estimate $T_{c}$ for a given compound with a nominal value of $n$ and under a given pressure $\mathrm{P}$, we perform an expansion of $T_{c}(n, P)$ in terms of $\mathrm{P}$. With the assumption of the linear variation of $n$ and $T_{c}^{\max }$ (or $\left.\Delta(0)\right)$ on the pressure, we obtain only terms up to third order, that is,

$$
T_{c}(n, P)=\sum_{Z=0}^{3} \alpha_{Z} P^{Z} / Z \text { ! }
$$

with

$$
\alpha_{Z}=\left(\frac{\partial}{\partial \Delta(0)} \frac{\partial \Delta(0)}{\partial P}+\frac{\partial}{\partial n} \frac{\partial n}{\partial P}\right)^{Z} T_{c}(n(P), \Delta(0, P)), z=1,2 \text { and } 3
$$

and $\alpha_{0}=T_{c}(n(0), \Delta(0))$ is just the $P=0$ term and where the derivatives $\frac{\partial \Delta(0)}{\partial P}$ and $\frac{\partial n}{\partial P}$ are determined by comparing with the experimental data; $\frac{\partial \Delta(0)}{\partial P}$ can be estimated by a set of values $T_{c} \times P$ for a given density $n$ and $\frac{\partial n}{\partial P}$ can be determined by Hall coefficients or thermoelectric power measurements. After determined these parameters, one can derive analytical expressions for each coefficient as function of $n$; using the universal parabolic fitting we can obtain a analytical expression for $\partial T_{c} / \partial n$ and studying the phase diagram for different values of $\Delta(0)$, we can calculate $\partial T_{c} / \partial P$ which calculates the $T_{c}$ dependency on $\mathrm{P}$ (assumption ii). This procedure gives an intrinsic term which has a clear interpretation since it comes from the changes in $\Delta(0)$ as well as a new third order term. It is remarkable that with a single set of parameters calculated for HG1201, with just the change in $T_{c}^{\max }$, we were also able to apply the same expansion (with same coefficients) to HG1212 and HG1223. 


\section{Comparison with the Exper- iments}

In order to apply the entire approach described above, we start with the $P=0$ curve $T_{c} \times n$ for $\mathrm{Bi}_{2} \mathrm{Sr}_{2} \mathrm{CaCu}_{2} \mathrm{O}_{8+y}$ which allows us to determine the initial parameters $b$ and $\Delta(0)$. We have used the values $t=0.05 \mathrm{eV}$ and $\mathrm{V}=0.052 \mathrm{eV}$ given in Ref.[7]. The coherence length estimate for $\mathrm{Bi}_{2} \mathrm{Sr}_{2} \mathrm{CaCu}_{2} \mathrm{O}_{8+y}$ is $\approx 13 \AA$ which is about $10 \%$ less than the $\xi$ for $\mathrm{YBaCuO}[11]$. Since we have used $b=2-3$ for fitting the phase diagram for these compounds, we use here $b=2.6-2.7$ in order to obtain $T_{c} \times n$ for $\mathrm{Bi}_{2} \mathrm{Sr}_{2} \mathrm{CaCu}_{2} \mathrm{O}_{8+y}$. The results are displayed on Fig.1 and one can see that it provides a very reasonable fitting for the experimental points of Allgeier et al[13]. In order to match the $T_{c}^{\text {max }}=92.5$ with $\mathrm{V}=0.052 \mathrm{eV}$, we used $\Delta(0)=214 \mathrm{~K}$ which is the same for all values of $n$. This value is very close $\Delta(0)=210 \mathrm{~K}$ used to the $\mathrm{Hg} 1210$ compounds because both compounds have very closed values for $T_{c}^{\max }=92-94 \mathrm{~K}$ and we used for both a $s_{x^{2}+y^{2}}$ gap function. We have also plotted a parametric curve which has been applied to fit the experimental points, $T_{c}=T_{c}^{\max }\left[1-\eta\left(n-n_{o p}\right)^{2}\right]$ with $T_{c}^{\max }=92.5 \mathrm{~K}$ and $\eta=9.0$. However the data of Huang et al[14] appears to have smaller $T_{c}^{\max }$, displaced toward higher hole densities and they are fitted with $\eta=25.0$. The parabolic is a pure phenomenological fitting which is used here only because it provides a analytical expression that can be used in the pressure expansion to estimate $\partial T_{c} / \partial n$.

Now that $b$ and $\Delta(0)$ has been determined let us study the $\mathrm{Bi}_{2} \mathrm{Sr}_{2} \mathrm{CaCu}_{2} \mathrm{O}_{8+y}$ compound under applied pressure. From the analysis of the phase diagram[13] shown in Fig. 1 and by varying the value of $\Delta(0)$ around the $P=0$ value of $\Delta(0)=214 \mathrm{~K}$, we can infer that $\partial T_{c} / \partial \Delta(0)=0.35$ at $n \approx 0.25$ which is near the optimal doping value of hole content used by Angilella et al[7]. From the Hall coefficient measurements[14], we get $d(\ln (n)) / d P=+8 \%$ and since their results seem to be shifted to larger values of hole contents, we estimate $d n / d P \approx 3.0 \times 10^{-2}$ which is two order of magnitude higher than previously derived $d n / d P \approx 1.8 \times 10^{-3}$ for the pressure effects on $\mathrm{Hg}$ compounds[12]. From the low pressure data we can get $\alpha_{1}=6.0 \mathrm{~K} / \mathrm{GPa}$ which allows us to derive the value $\partial \Delta(0) / \partial P=4.86 \mathrm{~K} / G P a$ which is necessary to determine all the high order coefficients. We noticed that this value is very close to $\partial \Delta(0) / \partial P=4.30 \mathrm{~K} / \mathrm{GPa}$ derived for the $\mathrm{Hg}$-based HTSC. Now, with the values of $\partial \Delta(0) / \partial P$ and $d n / d P$ determined, we can calculate the high order terms of Eq. 8 and in particular, we obtain $\alpha_{2}=-2.48 K / G P a^{2}$.
The third order coefficient was also calculated but it does not give any appreciable contribution in the range of pressure below $2 G P a$. Thus with all the coefficients of Eq. 8 determined, we are all set to calculate the values of $T_{c}(n, P)$. The calculated results are in excellent agreement with the experimental data[14] as it is shown in Fig.2. The large value of $d n / d P$ yields a very large negative second order coefficient $\alpha_{2}$ what makes the curve change its derivative at $P \approx 1.2 \mathrm{GPa}$. For the $\mathrm{Hg}$ compounds the change in sign of $d T_{c} / d P$ occurs at much larger $P \approx 30.0 \mathrm{GPa}$ and from the above analysis we can see that this difference in behavior is due to the large difference on the value of the charge transfer $d n / d P$ for this two compounds.

\section{Conclusions}

Thus, we conclude this work pointing out that our novel calculations based on a BCS type mean field and on the extended Hubbard Hamiltonian with the position of $\mathrm{V}$ as a variable parameter, is appropriate to describe the interplay between the $T_{c}$ and hole content for HTSC. The change in the position of the attractive interaction provides informations on the range of the Cooper pair attractive mechanism. Our method yields also a novel and systematic way to study the effects of the pressure and gives indication that the pressure induces a variation on the attractive potential which in turn, gives an intuitive interpretation on the origin of the intrinsic term. We should also emphasize that our procedure is based and has its starting point on a microscopic Hamiltonian and it differs from some pure phenomenological ways to fit the data[22] that uses a parabolic fitting to the experimental pressure data and are in the same category of the inverse parabolic fitting for the $T_{c} \times n$ curves mentioned above that are useful to obtain information on the different sets of data but has not any microscopic implications which is our main concern here. By applying it to the $\mathrm{Bi}_{2} \mathrm{Sr}_{2} \mathrm{CaCu}_{2} \mathrm{O}_{8+y}$ superconductors and comparing the results with our previous calculations on $\mathrm{Hg}$-based HTSC we have seen that the method not only deals successfully with the the experimental results but also displays clearly the physical properties that accounts for their different experimental behavior under pressure. 


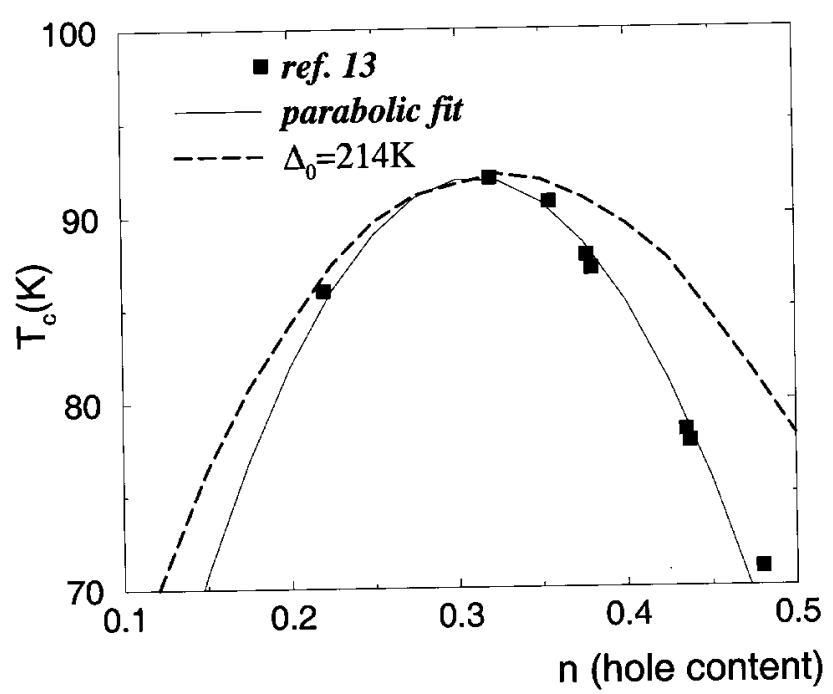

Figure 1. Calculations for the Bi phase diagram. The continuous line is the phenomenological fitting and the dashed line are the calculations described in the article. The squares are experimental points taken from Ref.13.

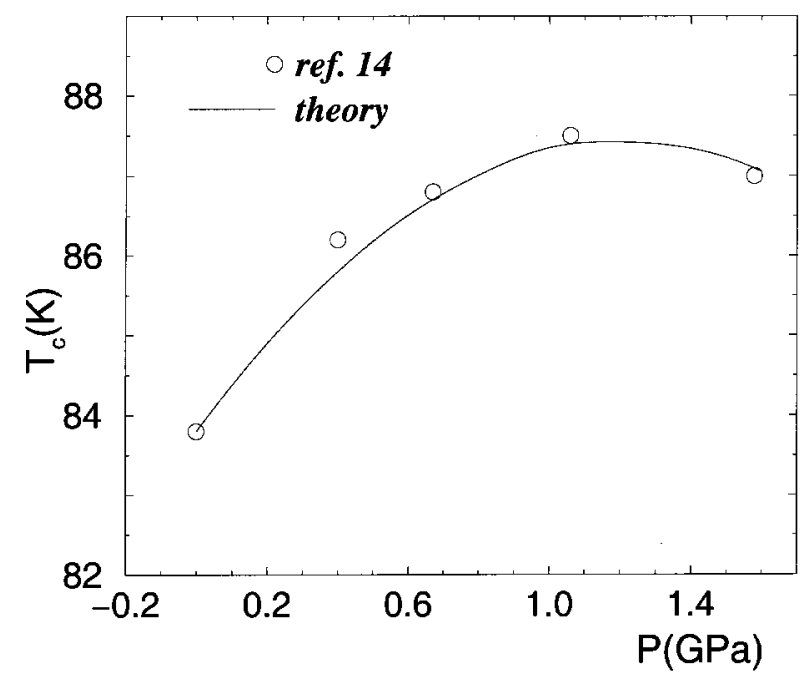

Figure 2. Calculations for the variations of $T_{c}$ for the Bi compound under pressure. The circles are experimental points taken from Ref.14.

\section{Acknowledgments}

We acknowledge partial financial support from the Brazilian agencies Capes and CNPq.

\section{References}

[1] L.Gao et al, Phys. Rev. B 50, 4260 (1994).
[2] R.J. Wijngaarden and R. Griessen, High Pressures Studies in Studies of High Temperature Superconductors, vol.2, p.29 edited by A.V. Narlikar (Nova Science, N.Y.,1989).

[3] H. Takahashi and N.Mori, in Studies of High Temperature Superconductors, vol.16, edited by A.V. Narlikar, (Nova Science, N.Y.,1996).

[4] J.S. Schilling and S. Klotz, in Physical Properties of High Temperature Superconductors, edited by D.M. Ginsberg, vol.II, (World Scientific, Singapore 1992).

[5] E.V.L. de Mello, Physica C 259, 109 (1996) and Check. J. Phys. 46, 945 (1996).

[6] R. Micnas, J. Ranninger and S. Robaszkiewicz, Rev. Mod. Phys. 62, 113 (1990).

[7] G.G.N. Angilella, R. Pucci and F. Siring, Phys. Rev. B 54, 15471 (1996).

[8] E.V.L. de Mello, Physica C, in press.

[9] Mécanique Quantique, L. Landau and I. Lifshitz, (Ed. Mir, Moscow 1966), page 190.

[10] P. Monthoux, A. V. Balatsky and D. Pines, Phys. Rev. Lett. 67, 3448 (1991).

[11] M. Cyrot and D. Pavuna, Introduction to Superconductivity and High-Tc Materials, World Scientific, Singapore (1992).

[12] E.V.L. de Mello and C. Acha, Phys. Rev. B 56, 466 (1997). and Physica C 282-287, 1819, (1997).

[13] C. Allgeier and J. S. Schilling, Physica C 168, 499 (1990).

[14] T. Huang et al, Phys. Rev. B 48, 7712 (1993).

[15] P.G. de Gennes, Superconductivity of Metals and Alloys, W.A. Benjamin, New York, 1966.

[16] R.J. Wijngaarden, E.N. Van Eenige, J.J. Scholtz and R. Griessen, Physica C 185-189, 787 (1991).

[17] C.C. Almasan, Phys. Rev. Lett. 69, 680 (1992).

[18] J.J. Neumeier and H.A. Zimmermann, Phys. Rev. B 47, 8385 (1993).

[19] R.P. Gupta and M. Gupta, Phys. Rev. B 5111760 (1995).

[20] M. Kosuge et al, Phys. Rev. B 45, 10713 (1992).

[21] Y. Cao, Q. Xiong, Y.Y. Xue and W. Chu, Phys. Rev. B 52, 6854 (1995).

[22] D. Tristan Jover, H. Wilhelm, R.J. Wijngaarden and R.S. Liu, Phys. Rev. B 55, 11832 (1997). 